\title{
A Face Recognition Method \\ Based on Total Variation Minimization and Log-Gabor Filter
}

\author{
Qingwen $\mathrm{Yu}^{\mathrm{a}}$, Xiaoyu Tan ${ }^{\mathrm{b}}$, Hong Wang ${ }^{\mathrm{c}}$ \\ School of Mechanical Engineering and Automation, Northeastern University, \\ Shenyang 110819, China. \\ aqwyu@mail.neu.edu.cn, ${ }^{b} 437156388 @ q q . c o m,{ }^{c}$ hwang@mail.neu.edu.cn
}

\begin{abstract}
Keywords: feature extraction; total variation minimization; Log-Gabor filter; face recognition Abstract. A new face recognition method is proposed by using total variation minimization and Log-Gabor filter. First of all, facial images are transformed by total variation minimization model. Secondly, the facial feature is extracted by Log-Gabor filter from the result of the former transformation. Then, dimensionality reduction is realized by using principle component analysis. Finally, classification is achieved by using nearest neighbor classifier. This method is a kind of combination of advantage between total variation minimization and Log-Gabor filter which holds edge feature and describes textural feature of facial image reasonably. The experiment is conducted on Yale Face database. Compared with the methods such as Gabor filter based feature extraction, et al., the face recognition method we presented has better recognition performance. The correct recognition rate reaches to $86.36 \%$.
\end{abstract}

\section{Introduction}

Face recognition has been a hot topic in biometric research nowadays, since it has a very wide range of applications, such as in law enforcement for mugshot identification, in personal identification for driver's licenses and in public security systems [1]. In face recognition procedure, facial feature extraction is an important step. By using various feature extraction methods, the performance of face recognition systems has steadily improved in recent years. Compared with other feature extraction methods, Gabor filter has remarkable mathematical and biological properties, so it has been successfully applied to feature extraction task [2,3]. However, Gabor filter presents some limitations: Firstly, the maximum bandwidth of a Gabor filter is limited to about one octave. Secondly, there's a nonzero DC component in the even-symmetric filter for wide bandwidths [4]. Thus, Gabor filters are not optimal for broad spectral information with maximal spatial localization.

Given this situation, this paper presents an efficient method based on total variation minimization and Log-Gabor filter for facial feature extraction. Total variation minimization can preserve edge details of the image, while removing noise. Log-Gabor filter has an extended tail at the high frequency end and it has no DC component, so it can better represent the frequency properties of natural images. Thus, the method can describe the edge feature and the textural feature of a facial image reasonably.

To test the performance of the method, we conduct experiments on Yale Face Database, the classification is based on nearest neighbor classifier. The results show the effectiveness of the proposed method.

\section{Description of total variation minimization}

Total variation minimization most often used in digital image processing has applications in noise removal and image restoration [5,6]. Usually, an observed image $I$ can represented as a function as follows,

$$
I=H^{*} f+s,
$$


where $f$ is the ideal original image, $H$ is point spread function and $s$ is an additive Gaussian noise with zero mean and standard deviation $\sigma$. Recovering $f$ from $I$ is an ill-posed inverse problem and it can be solved via total variation minimization model. The total variation minimization model of Eq. 1 can be written in the following form:

$$
\underset{f}{\arg \min } \mu\|H * f-I\|_{1}+\|f\|_{T V},
$$

where $\mu$ is a scalar threshold on scale and

$$
\|f\|_{T V}=\sum_{i}\left(D f_{i}\right)
$$

which is the total variation. Here, $D f_{i}$ is the first order forward finite-difference operator along the horizontal and vertical directions of the $i$ th component of $f$. Eq. 2 can be solved by introducing two intermediate variables $v$ and $u$,

$$
\begin{aligned}
& \underset{f, u, v}{\arg \min } \mu\|v\|_{1}+\|u\|_{1} \\
& \text { s.t.v }=H^{*} f-I, u=D f .
\end{aligned}
$$

Then the idea of the augmented Lagrangian method is used to solve Eq. 4 as below,

$$
\begin{gathered}
f_{k+1}=\underset{f}{\arg \min } \frac{\alpha}{2}\left\|v_{k}-H^{*} f_{k}+I\right\|^{2}+\frac{\beta}{2}\left\|u_{k}-D f_{k}\right\|^{2}+x_{k}{ }^{T} H^{*} f_{k}+y_{k}{ }^{T} D f_{k}, \\
u_{k+1}=\underset{u}{\arg \min }\left\|u_{k}\right\|_{1}-y_{k}^{T}\left(u_{k}-D f_{k+1}\right)+\frac{\beta}{2}\left\|u_{k}-D f_{k+1}\right\|^{2}, \\
v_{k+1}=\underset{v}{\arg \min } \mu\left\|v_{k}\right\|_{1}-x_{k}{ }^{T} v_{k}+\frac{\alpha}{2}\left\|v_{k}-H^{*} f_{k+1}+I\right\|^{2},
\end{gathered}
$$

where $x$ and $y$ are Lagrange multipliers, $\alpha$ and $\beta$ are regularization parameters. By updating the Lagrange multipliers as follows, the above three problems can be solved with iterative process.

$$
\begin{gathered}
x_{k+1}=x_{k}-\alpha\left(v_{k+1}-H^{*} f_{k+1}+I\right), \\
y_{k+1}=y_{k}-\beta\left(u_{k+1}-D f_{k+1}\right) .
\end{gathered}
$$

Since total variation minimization model is effective for image denoising with edge preservation, it is used here for face image processing. The face image in face database is the observed image $I$. After using total variation minimization model, the transformed image $f$ is got. Fig. 1 shows the cropped Yale faces and their transformed images. 


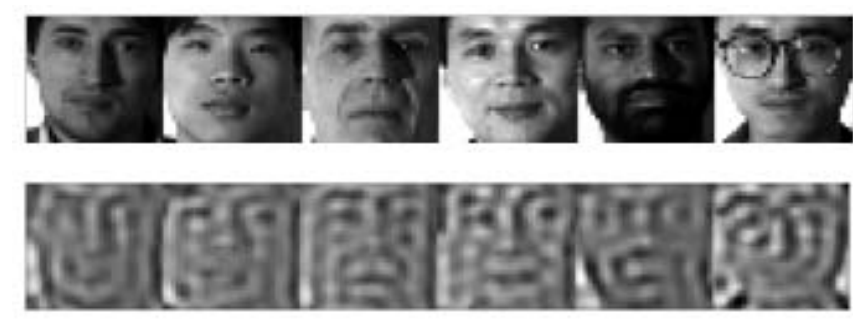

Fig.1. The cropped Yale face images and the transformed images

\section{Log-Gabor Feature Representation}

Log-Gabor filters are proposed by Field [4]. According to Field, when viewed on a logarithmic axis, Log-Gabor filters have Gaussian transfer functions. These are as symmetric as frequency-response functions of many cortical cells. Also Log-Gabor transform has an extended tail and has no DC component, so they allow arbitrarily large bandwidth filters to be constructed, while Gabor transform would over-represent the low frequency components and under-represent the high frequency components. Some measurements of the characteristics of mammalian visual cortical cells have been made by a number of researchers, and they found that our spatial filters of bandwidths ranging from about 0.5 octaves up to about 3 octaves $[7,8]$. Therefore, Log-Gabor is preferred to encode the natural images.

In frequency domain 2D Log-Gabor filters have two components. One is the radial component $G(f)$ which controls the bandwidth that the filter responds to and the other is the angular component $G(\theta)$ which controls the orientation selectivity of the filter. They can be defined as follows,

$$
G(f, \theta)=G(f) \times G(\theta)=e^{-\frac{\left(\log \left(f / f_{0}\right)\right)^{2}}{2\left(\log \left(\sigma_{f} / f_{0}\right)\right)^{2}}} \times e^{\frac{-\left(\theta-\theta_{\theta}\right)^{2}}{2 \sigma_{\theta}^{2}}},
$$

where $f_{0}$ represents the center frequency, $\sigma_{f}$ is the scaling factor of the filter bandwidth, $\theta_{0}$ is the orientation angle, and $\sigma_{\theta}$ defines the angular bandwidth. In spatial domain 2D log-Gabor filters also have two components, the even-symmetric component and odd-symmetric component, as shown in Fig. 2.
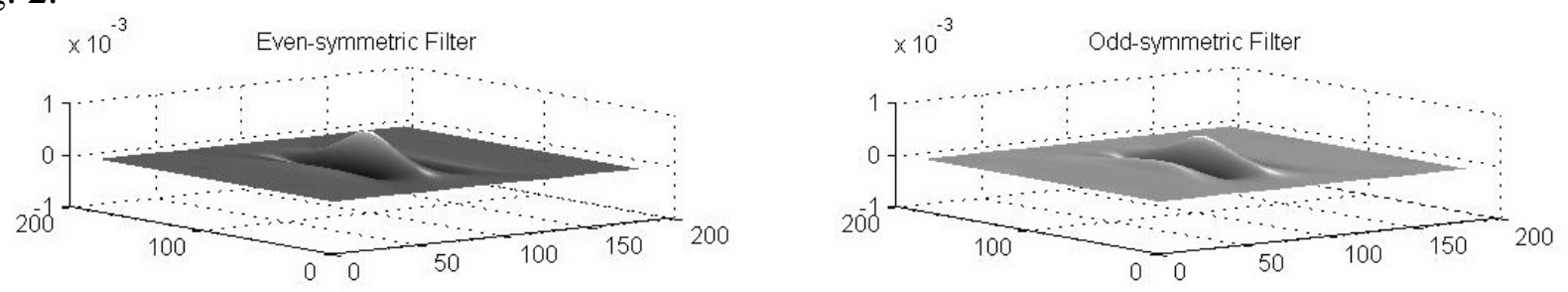

Fig. 2. 2D log-Gabor filters seen in spatial domain

To construct a Log-Gabor feature, the image should be filtered with a bank of Log-Gabor wavelets in the frequency domain. First, compute the Fast Fourier Transform of the image. Next, convolve the transformed image with the initially designed Log-Gabor wavelets of $s$ different scales and $o$ orientations. Then, transfer the spectra back to the spatial domain by inverse Fast Fourier Transform, and obtain the amplitudes of filtered image. Finally, each image will have $s \times o$ filtered representations. Taking an image $I$ for example, each of its $s \times o$ Log-Gabor feature representations $L$ will be

$$
L(s, o)=\operatorname{abs}(\operatorname{IFFT} 2(G(s, o) * F F T 2(I))) .
$$

Fig. 3 illustrates a facial image in our data set and its response to Log-Gabor filters at 5 scales and 8 orientations. 


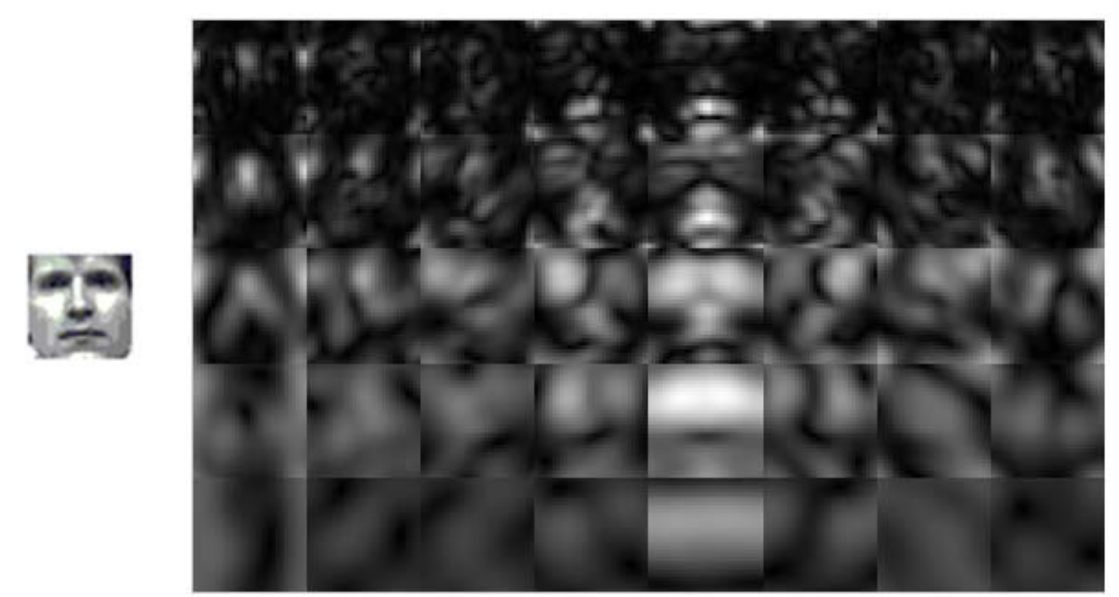

Fig. 3. A facial image and its response to Log-Gabor filters at 5 scales and 8 orientations

\section{Experiment and Result}

Yale face database [9] is employed in the experiment. The database contains 165 grayscale images for 15 subjects of different races and features (glasses, moustache, etc.), with 11 images per person. The images contain different facial expressions, views and illumination conditions for each subject. The image size is $243 \times 320$ pixels. Randomly divides the original data into two subsets: a training set and a testing set. 5 images per person, totally 75 images were chosen randomly to form the training set. The remaining 90 images were used to form the testing set. This was repeated 50 times, and all the results reported below were averaged over the 50 runs. All the image data used here are manually aligned, cropped, and then re-sized to 32x32 images.

Firstly, total variation minimization algorithm was applied to face data. Figure 1 shows the results of total variation minimization methods on some cropped images. Then, a set of Log-Gabor filters with 5 scales and 8 orientions were used on the results of last step to extract the facial feature.Thus, each face data is of $32 \times 32 \times 40$ dimensions. It means a large amount of computation and memory cost. Hence, it is sensible to reduce the features dimensions by a simple and efficient method. Here we use downsampling to obtain 2,560 dimensions. After further dimensionality reduction with principle component analysis (PCA), the face data was classified by nearest neighbor classifier.

To validate the accuracy of the proposed method, here we also do the experiments with PCA method, Gabor Filter-based PCA method and Log-Gabor filter-based PCA method. Since Median filtering(MF) is a kind of technique which is effective at removing noise and preserving edges, the experiment with MF and Log-Gabor filter is also conducted.

The experimental result is shown in Fig. 4. Table 1 illustrates the maximum recognition rate of each menthod. The results show that the methods based on Log-Gabor filter have better recognition performance than Gabor-based method and the method based on total variation minimization is more conducive to improving the recognition rate than the method based on MF. Hence, the method we present based on total variation minimization and Log-Gabor filter can represent facial feature more effectively with lower feature dimension.

Table 1 Maximum recognition rates of all the methods

\begin{tabular}{c|c|c}
\hline Method & Max Recognition rate[\%] & Feature dimension \\
\hline PCA & 72.73 & 40 \\
Gabor+PCA & 80 & 75 \\
Log-Gabor+PCA & 83.64 & 30 \\
TV+Log-Gabor+PCA(Ours) & 86.36 & 30 \\
MD+Log-Gabor+PCA & 79.09 & 40 \\
\hline
\end{tabular}




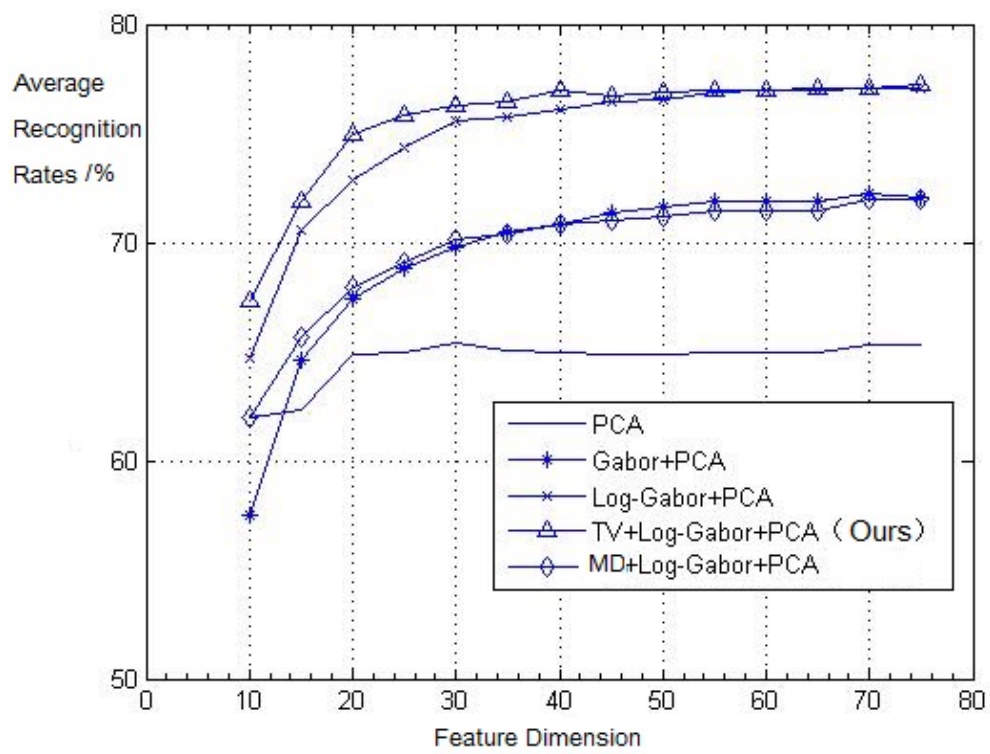

Fig. 4. The average recognition rates with respect to the feature dimension

\section{Conclusion}

In this paper, we present a novel method for face recognition based on total variation minimization and Log-Gabor filter. The facial fearture extracted with total variation minimization can preserve edge very well. Then, Log-Gabor filter can better represent the frequency properties of the face images. The experiment results show that the method can efficiently encode human face and it has good performance.

\section{Acknowledgements}

This work was financially supported by National Natural Science Foundation of China (61071057, 51405073), the University Innovation Team of Liaoning Province (LT2014006) and the State Key Laboratory of Process Industry Automation of China (PAL-N201304).

\section{References}

[1] Zhao W, Chellappa R, Philips P, et al. Face recognition: A literature survey [J]. ACM Computing Surveys, 2003, 35(4): 399-458.

[2] Zhang B, Shan S, Chen X, et al. Histogram of Gabor Phase Patterns (HGPP): A novel object representation approach for face recognition [J]. IEEE Transactions on Image Processing, 2007, 16(1): 57-68.

[3] Zheng Z, Yang F, Tan W, et al. Gabor feature-based face recognition using supervised locality preserving projection [J]. Signal Processing, 2007, 87(10): 2473-2483.

[4] Field D. Relations between the statistics of natural images and the response properties of cortical cells [J]. Journal of the Optical Society of America, 1987, 4 (12): 2379-2394.

[5] Chan S, Khoshabeh R, Gibson K, et al. An augmented Lagrangian method for total variation image restoration. IEEE Transactions on Image Processing, 2011, 20(11): 3097-3111.

[6] Zhao Xiao-fei, Zhang Hong-zhi, Zuo Wang-meng, et al. Survey of augmented Lagrangian methods for total variation-based image restoration [J]. Intelligent Computer and Applications, 2012, 2(3): 44-47, In Chinese. 
[7] Kovesi P. Invariant measures of image features from phase information[D].Perth: University of Western Australian, 1996.

[8] Wang W, Li J, Huang F, et al.Design and implementation of Log-Gabor filter in fingerprint image enhancement[J].Pattern Recognition Letters, 2008, 29(3): 301-308.

[9] Bellhumer P., Hespanha J., Kriegman D.. Eigenfaces vs. fisherfaces: Recognition using class specific linear projection[J]. IEEE Transactions on Pattern Analysis and Machine Intelligence, Special Issue on Face Recognition, 1997, 17(7): 711-720. 